\title{
Estilo de Apego, Empatía y Autoestima en Agresores de Pareja
}

\section{Attachment Style, Empathy and Self-Esteem in Partner-Violent Men}

\author{
Ismael Loinaz \\ Universidad del País Vasco UPV/EHU, España \\ Enrique Echeburúa \\ Universidad del País Vasco UPV/EHU \\ Cibersam, España \\ $\&$ \\ María Ullate \\ Universidad del País Vasco UPV/EHU, España
}

(Rec: 15 de marzo de 2012 / Acep: 2 de mayo de 2012)

\begin{abstract}
Resumen
Frecuentemente se señalan variables emocionales para describir el perfil de los agresores de pareja y para incorporarlas a los programas de tratamiento. El presente estudio compara las puntuaciones en apego adulto, empatía y autoestima de 90 agresores de pareja en prisión con un grupo de control de la población general $(\mathrm{N}=94)$. Ambas muestras fueron evaluadas con el Cuestionario de Apego Adulto, el Índice de Reactividad Interpersonal y la Escala de Autoestima. Los agresores fueron clasificados en dos conglomerados para valorar la posible existencia de un subtipo patológico y otro parecido a la población general. Los resultados no muestran diferencias significativas entre los grupos. Pese a no confirmarse las hipótesis predominantes a nivel académico, los resultados son congruentes con estudios recientes que señalan la ausencia de diferencias entre agresores y grupos normativos. Se discute el efecto de los instrumentos de evaluación en los resultados y su utilidad para las intervenciones.
\end{abstract}

Palabras clave: Agresores de pareja, apego adulto, empatía, autoestima

\begin{abstract}
It is common to refer emotional variables to describe the profile of partner-violent men as well as to include them in treatment programs. This study compares scores on adult attachment, empathy and self-esteem of 90 offenders in prison and a control group from the general population $(\mathrm{N}=94)$. Both samples were assessed with the Adult Attachment Questionnaire, the Interpersonal Reactivity Index and the Rosenberg Self-Esteem Scale. The offenders were also classified into two clusters to assess the possible existence of a pathological subtype and other similar to the control group. The results show no significant differences between groups. Although the prevailing hypothesis in the academic context has not been confirmed, the results are consistent with recent studies that indicate no differences between aggressors and control groups. The effect of the assessment tools on the results and their usefulness for interventions are discussed.
\end{abstract}

Keywords: Partner-violent men, adult attachment, empathy, self-esteem

Parte del estudio ha sido posible gracias a las ayudas económicas concedidas al primer autor por el Centro de Estudios Jurídicos y Formación Especializada (Departament de Justicia, Generalitat de Catalunya; Proyecto SC-3.167.10) y del Gobierno Vasco (Programa de Formación de Personal Investigador; Proyecto BFI-09.185). Este estudio ha sido apoyado por el Grupo Consolidado de Investigación en Psicología Clínica del Gobierno Vasco y por la UFI 11/04 de la Universidad del País Vasco UPV/EHU.

Correspondencia: La correspondencia relacionada con este artículo debe ser dirigida a Ismael Loinaz, Universidad del País Vasco UPV/EHU, Facultad de Psicología - Departamento de Personalidad, Evaluación y Tratamiento Psicológico. Avda. de Tolosa, 70. 20018 San Sebastián (España). E-mail: ismael. loinaz@gmail.com 


\section{Introducción}

El componente emocional puede considerarse un factor con gran peso en el análisis de la conducta violenta (Shaver \& Mikulincer, 2011). En el caso de la violencia contra la pareja, es frecuente el uso de dimensiones afectivas en la clasificación de los agresores, con descriptores como emocionalmente inestables (Dutton \& Golant, 1997; Loinaz, Echeburúa \& Torrubia, 2010), volátiles (Saunders, 1992), cíclicos (Dutton, 1988) o disfóricos (Holtzworth-Munroe \& Stuart, 1994). Entre las variables emocionales relacionadas con la conducta violenta desempeñan un papel importante el apego, la empatía y la autoestima (Hollin, 2003; Jones, 2008; McMurran \& Howard, 2009; Renzetti \& Edleson, 2008).

La incorporación del apego al análisis de la etiología de la violencia ha permanecido en segundo plano hasta fechas muy recientes (Mauricio \& Gormley, 2001). Sin embargo, en los últimos años se encuentran a nivel internacional múltiples referencias que vinculan el apego adulto a la conducta violenta (Ansbro, 2008; Beech \& Mitchell, 2009; Forbes \& Reilly, 2011; Meloy, 2003; Mikulincer \& Shaver, 2011; Ross \& Pfäfflin, 2007). Pese a que en el contexto español se han invertido algunos esfuerzos en su estudio (AlonsoArbiol, Balluerka \& Shaver, 2007; Alonso-Arbiol, Shaver \& Yarnoz, 2002; Melero \& Cantero, 2008), la investigación en esta área aún está por desarrollarse, en especial en lo referente a su relación con la conducta violenta, donde solo una investigación incluye esta variable (Loinaz, 2011a, b).

La teoría del apego ofrece información relevante sobre los estilos relacionales de los agresores y el modo en que las interacciones violentas se producen. Los procesos de apego podrían influir sobre nuestra expresión funcional o disfuncional de la ira, la violencia doméstica y de pareja, el comportamiento antisocial, o incluso la violencia entre grupos (Mikulincer \& Shaver, 2011). El apego no explica la conducta, pero sí ofrece un método de análisis sobre los procesos psicológicos que median en la agresión, tales como ciertos déficits cognitivos o emocionales (falta de empatía o tendencia a la impulsividad) (Ansbro, 2008).

Según (Dutton, 2008, 2011), la agresión en la pareja puede entenderse como un comportamiento de protesta dirigido a la figura de apego y precipitado por amenazas de separación o abandono. A nivel internacional, la variable del apego forma parte de los protocolos para evaluar a los hombres violentos con la pareja (Fournier, Brassard \& Shaver, 2011), así como para diferenciar subtipos de agresores (Amor, Echeburúa \& Loinaz, 2009; Babcock, Jacobson, Gottman \& Yerington, 2000; Dutton, 2007; Holtzworth-Munroe \& Meehan, 2004; HoltzworthMunroe, Meehan, Herron, Rehman \& Stuart, 2000, 2003; Holtzworth-Munroe \& Stuart, 1994; Holtzworth-Munroe, Stuart \& Hutchinson, 1997; Lawson, 2008; Mauricio \& López, 2009) o valorar resultados terapéuticos (Lawson, Barnes, Madkins \& Francois-Lamonte, 2006; Lawson \& Brossart, 2009).
A grandes rasgos, los agresores de pareja, en comparación con sujetos no violentos, muestran apego inseguro, poca estabilidad emocional y gran ansiedad frente al rechazo o abandono por parte de la pareja (Babcock et al., 2000; Dutton, Saunders, Starzomski \& Bartholomew, 1994; Holtzworth-Munroe et al., 1997; Lawson, 2008; Mayseless, 1991; Tweed \& Dutton, 1998). Este apego inseguro se relaciona con una mayor necesidad de dominio en las relaciones íntimas (Mauricio \& Gormley, 2001) y con una especial vulnerabilidad a los sentimientos de abandono (Mikulincer \& Shaver, 2011).

Por otro lado, el apego ansioso (preocupado y temeroso) se ha relacionado con la agresión a la pareja en numerosos estudios (Fournier et al., 2011; Lawson \& Brossart, 2009; Mauricio \& López, 2009; Mikulincer \& Shaver, 2007). La asociación de estilos de apego inseguros u hostiles con variables negativas como una menor autoestima, mayor impulsividad o mayor ira también ha sido encontrada en una muestra española de agresores de pareja (Loinaz, 2011a, b).

Otra variable emocional que podría desempeñar un papel relevante en la conducta violenta es la empatía. Sus déficits han sido identificados como un importante antecedente y facilitador de la conducta agresiva, mientras que su funcionamiento normal se contempla como un factor protector que inhibe la agresión y motiva al altruismo (Day, Casey \& Gerace, 2010; Day, Mohr, Howells, Gerace \& Lim, 2011; Jolliffe \& Farrington, 2004, 2007; Palmer, 2005). En este sentido, si un sujeto es capaz de empatizar y, por tanto, de experimentar las consecuencias de sus actos sobre los demás, será menos probable que dañe a otros y será más propenso a ayudarles (McPhedran, 2009).

La investigación actual apoya la visión de la empatía como un constructo multi-componente, formado por aspectos cognitivos (reconocer e identificarse con los sentimientos del otro) y emocionales (experimentar dichos sentimientos) (Day et al., 2010; Fernández-Pinto, López-Pérez \& Márquez, 2008; Marshall, Marshall, Serran \& O’Brien, 2009; McPhedran, 2009; Owen \& Fox, 2011). Según Davis (1996), la empatía está compuesta por cuatro constructos: antecedentes (aspectos del observador, del receptor y de la situación), procesos (medios para generar una respuesta empática, cognitivos o no), respuestas intrapersonales (reacciones cognitivas y emocionales del observador, resultado de la exposición al objetivo) y respuestas interpersonales (acciones dirigidas hacia el objeto de la empatía, incluida la conducta de ayuda o la agresión). Estos aspectos son evaluados por el Índice de Reactividad Interpersonal (Davis, 1980). Sin embargo, la respuesta empática no es igual en todos los sujetos ni en todas las situaciones y, en ocasiones, los distintos procesos y consecuencias de los mismos pueden derivar en una conducta violenta o de distanciamiento. Así pues, las diferencias individuales y situacionales explican por qué los déficits en empatía son 
dependientes del contexto o de la víctima más que un rasgo fijo (Day et al., 2011; Fernández-Pinto et al., 2008).

La empatía tiene un gran peso en el ámbito rehabilitador. Su mejora es un componente central en los programas diseñados para intervenir con agresores, si bien hay pocas pruebas que permitan valorar su efecto en la reducción del riesgo de reincidencia (Day et al., 2010). Respecto a la violencia en la pareja, son escasos los estudios que analizan específicamente su rol en los agresores (Covell, Huss \& Langhinrichsen-Rohling, 2007), pese a que su abordaje forme parte de los módulos de entrenamiento en habilidades sociales y resolución de problemas, incremento de conciencia y mejora de la motivación para el cambio (Bowen, 2011; Dobash, Dobash, Cavanagh \& Lewis, 2000; Gondolf, 2002; Millana, 2011). En nuestro contexto la variable se ha evaluado como parte de los programas de tratamiento y el análisis de su eficacia (Boira, López, Tomás \& Gaspar, 2010; Boira \& Tomás, 2011; Echeburúa \& FernándezMontalvo, 2009; Echeburúa, Fernández-Montalvo \& Amor, 2006; Echeburúa, Sarasua, Zubizarreta \& de Corral, 2009).

A nivel internacional, algunos estudios han descrito la existencia de diferencias en la respuesta empática entre subtipos de agresores de pareja (Holtzworth-Munroe \& Stuart, 1994; Tweed \& Dutton, 1998), pero también se ha demostrado que estas no son significativas tras la intervención (Huss \& Ralston, 2008). La existencia de variabilidad entre sujetos violentos y la población general parece más clara en muestras de jóvenes que de adultos (Jolliffe \& Farrington, 2004). Los resultados más recientes siguen siendo inconcluyentes a favor (Owen \& Fox, 2011) o en contra (Day et al., 2011) del peso de la ausencia de empatía como variable explicativa del comportamiento violento.

En cuanto a la autoestima, se ha asociado una baja autoestima con una mayor frecuencia y gravedad de las conductas violentas (Walker \& Bright, 2009). Sin embargo, las conclusiones resultan inconsistentes por la multidimensionalidad del constructo, las dificultades de su medición y por su asociación con distintos tipos de violencia (Bushman et al., 2009; Maples et al., 2010; Ostrowsky, 2010). Desde el punto de vista teórico es posible defender la relación de la conducta violenta tanto con una baja autoestima (a través de sentimientos de inferioridad, la culpabilización de los demás, etcétera) como con una autoestima elevada y rasgos narcisistas (arrogancia), sin que haya estudios empíricos que apoyen más ninguna de las opciones (Ostrowsky, 2010).

La relación baja autoestima/mayor violencia se ha confirmado en agresores de pareja en muestras forenses (Dick, 2004; Papadakaki, Tzamalouka, Chatzifotiou \& Chliaoutakis, 2009), pero no en muestras penitenciarias (Cale \& Lilienfeld, 2006). En nuestro contexto, por ejemplo, se han observado diferencias en autoestima entre distintos tipos de agresores de pareja (Loinaz, 2011a, b; Loinaz et al., 2010), pese a que de momento no se han asociado específicamente a un nivel de violencia en la Conflict
Tactics Scales-2 -CTS-2- (Loinaz, Echeburúa, Ortiz-Tallo \& Amor, 2012). Se debe tener en cuenta que la mayoría de los estudios que encuentran una alta relación entre baja autoestima y conducta violenta se han realizado con muestras de estudiantes (Walker \& Bright, 2009; Walker \& Knauer, 2011), lo que constituye una limitación cuando se pretenden extrapolar los resultados al contexto clínico, jurídico y forense.

Al igual que ocurre con el apego y la empatía, se ha señalado la necesidad de comprender la autoestima como un constructo multi-dimensional (Kirkpatrick, Waugh, Valencia \& Webster, 2002; Sakellaropoulo \& Baldwin, 2007; Sandstrom \& Jordan, 2008; Walker \& Bright, 2009; Webster, Kirkpatrick, Nezlek, Smith \& Paddock, 2007). Así pues, existiría una autoestima saludable pero también una autoestima falsamente inflada, relacionada con rasgos narcisistas y destructivos. Esta última está basada en tendencias egocéntricas y prepotentes que alimentan el sentimiento de superioridad o de poder sobre los demás y explican la relación existente con la violencia (Bushman et al., 2009; Maples et al., 2010; Taylor, Davis-Kean \& Malanchuk, 2007; Thomaes \& Bushman, 2011; Thomas, Bushman, Stegge \& Olthof, 2008). En cualquier caso, la relación entre autoestima y violencia no es del todo clara y, por ello, es necesario diferenciar esta emoción del narcisismo o de la arrogancia (Maples et al., 2010; Ostrowsky, 2010; Papadakaki et al., 2009; Walker \& Bright, 2009).

Ante la inconsistencia de los resultados obtenidos se considera de interés el análisis en profundidad de los factores emocionales (apego, empatía y autoestima) en los agresores como grupo, así como su comparación respecto a la población general y la descripción de posibles diferencias entre grupos de agresores. Todo ello puede contribuir a un mejor entendimiento de la etiología de la conducta violenta en la pareja. Las hipótesis de partida son las siguientes: 1) los agresores como grupo van a presentar un estilo afectivo diferente al del grupo de control, con una menor empatía, una autoestima más baja y un estilo de apego inseguro o ansioso; y 2) en el grupo de agresores será posible diferenciar distintos perfiles afectivos, existiendo subgrupos de agresores más semejantes al grupo de control y otros más patológicos.

\section{Método}

\section{Participantes}

Los participantes del grupo de agresores $(\mathrm{N}=90)$ estaban internados en el Centro Penitenciario Brians-2 (Barcelona) entre 2008 y 2010 por delitos relacionados con la violencia de género. Asimismo se ha creado un grupo de control ( $\mathrm{N}=94)$ con características socio-demográficas similares a las de los agresores para comparar las distintas medidas. Este grupo proviene de la población general y sus respuestas 
Tabla 1. Descriptivos sociodemográficos de las dos muestras

\begin{tabular}{llll}
\hline & \multicolumn{1}{c}{$\begin{array}{c}\text { Agresores } \\
(\mathrm{N}=90)\end{array}$} & $\begin{array}{c}\text { Grupo de control } \\
(\mathrm{N}=94)\end{array}$ & $p$ \\
\hline Edad & & & \\
$\mathrm{M}(\mathrm{DT})$ & $36.38(8.6)$ & $37.94(12.01)$ & 0.443 \\
Rango & $22-58$ & $18-60$ & \\
& & & \\
Nacionalidad & & & 0.020 \\
Españoles & $69 \%(62)$ & $16 \%(15)$ & \\
Extranjeros & $31 \%(28)$ & & \\
Nivel de estudios & & 34 & 0.074 \\
Primarios & 25.3 & 37.5 & \\
Secundarios & 57.5 & 21.9 & -0.19 \\
Formación profesional & 9.2 & 6.3 & \\
Universitarios & 8 & & \\
\hline
\end{tabular}

fueron recopiladas por la tercera firmante de este trabajo en su entorno comunitario. Las características demográficas de la muestra del grupo de agresores y del grupo de control figuran expuestas en la tabla 1.

\section{Instrumentos}

Cuestionario de Apego Adulto (CAA) (Melero \& Cantero, 2008). Se trata de un instrumento elaborado y baremado con una muestra española. Está compuesto por 40 ítems y ofrece información en cuatro escalas: Escala 1: baja autoestima, necesidad de aprobación y miedo al rechazo; Escala 2: resolución hostil de conflictos, rencor y posesividad; Escala 3: expresión de sentimientos y comodidad con las relaciones; Escala 4: autosuficiencia emocional e incomodidad con la intimidad. Este instrumento permite la clasificación de los sujetos en dos clusters (seguros/ inseguros) o en cuatro (temerosos, preocupados, seguros y alejados), según se combinen las cuatro escalas. Su consistencia interna oscila de 0.68 a 0.86 .

Índice de Reactividad Interpersonal (IRI) (Davis, 1980). Es una de las medidas más utilizadas para evaluar la empatía. La empatía está relacionada con la disposición prosocial y la inhibición de la agresividad (Mestre, Frías \& Samper, 2004). El índice está formado por 28 ítems que se puntúan en una escala tipo Likert (1-5) y evalúan cuatro dimensiones: 1) Toma de Perspectiva: mide la capacidad para apreciar el punto de vista de los demás y los intentos del sujeto por adoptar la perspectiva del otro ante situaciones de la vida cotidiana; 2) Fantasía: mide la capacidad imaginativa para ponerse en situaciones ficticias e identificarse con personajes ficticios; 3) Preocupación Empática: mide la capacidad para mostrar compasión, preocupación y cariño frente al malestar de otros (sentimientos orientados al otro); 4) Malestar Personal: evalúa sentimientos de ansiedad y malestar al observar experiencias negativas en los demás (sentimientos orientados al yo). En su versión original presenta una fiabilidad interna de 0.70-0.78 (Davis, 1980), mientras que la versión utilizada encuentra valores de 0.56-0.70 en las distintas escalas. La escala toma de perspectiva y la de preocupación empática son las que más correlacionan entre sí y constituyen los componentes más importantes de la respuesta empática. La escala ha sido utilizada en estudios previos con agresores de pareja en nuestro entorno, pero el formato de las puntuaciones (por diferencias en las versiones) no permite su comparación directa. La validez de constructo del instrumento muestra correlaciones entre los factores 1,2 y 3 y la conducta prosocial (correlaciones entre 0.151 y 0.314 para varones).

Escala de Autoestima (EA) (Rosenberg, 1965). Evalúa el sentimiento de satisfacción que una persona tiene consigo misma. Consta de 10 ítems con respuestas de tipo Likert (1-4). Se han utilizado los siguientes puntos de corte: 25 puntos, autoestima baja; 26-29, autoestima media; y 30, autoestima alta. En muestra clínica española (Vázquez, Jiménez \& Vázquez-Morejón, 2004), la escala obtiene una consistencia interna de 0.87 , una fiabilidad test-retest de 0.74 en un año y una validez de constructo adecuada con herramientas como el SLC-90 (-0.61 para la puntuación total y superiores a 0.60 en las escalas Sensibilidad Interpersonal y Depresión).

\section{Procedimiento}

Como parte de un protocolo de evaluación más amplio (ver Loinaz, 2010, 2011), la muestra de agresores de pareja respondió a las tres pruebas analizadas en el presente estudio en sesiones grupales tras haber sido entrevistados individualmente por el primer autor. Todos los sujetos participaron de forma voluntaria en el estudio y tras firmar un consentimiento informado. El grupo de control respondió exclusivamente a las tres pruebas y de forma específica para este estudio en 2011. Se les ofreció la devolución de los resultados para motivar su participación, así como la posibilidad de participar de forma anónima en el estudio. 


\section{Análisis de datos}

Todos los datos fueron analizados con el PASW 18 para Windows. Las muestras fueron comparadas con pruebas paramétricas ( $T$ de Student) y no paramétricas ( $U$ de Mann Whitney y Chi-Cuadrado), y se calculó el tamaño del efecto de las diferencias significativas ( $d$ de Cohen para variables cuantitativas y Phi para las distribuciones). La clasificación de los agresores en dos grupos se llevó a cabo mediante análisis de conglomerados (análisis de conglomerados jerárquicos, método Ward), tomando en consideración las puntuaciones obtenidas en los tres instrumentos.

\section{Resultados}

\section{Cuestionario de Apego Adulto (CAA)}

En la tabla 2 se presentan las puntuaciones medias obtenidas por la muestra de agresores y por el grupo de control en las cuatro escalas en las que se divide el CAA.
Las diferencias entre ambos grupos son solo significativas para la escala 1 (baja autoestima, necesidad de aprobación y miedo al rechazo) en la que puntúan más alto los sujetos del grupo de control. Pese a ello, la magnitud de la diferencia (d) es solo moderada.

El CAA permite hacer una corrección dimensional basada en los baremos obtenidos en la validación del instrumento (ver Melero \& Cantero, 2008). En nuestra muestra (ver tabla 3), los baremos no se ajustarían a las descripciones realizadas por los autores para la clasificación del apego en dos o cuatro dimensiones. Pese a ello, los agresores se ubicarían entre los estilos preocupado y alejado (para cuatro dimensiones) y seguro (para dos dimensiones). El grupo de control se ubica en unas puntuaciones moderadas en todas las escalas que imposibilitan esta clasificación.

\section{Indice de Reactividad Interpersonal (empatía)}

En la tabla 4 se comparan las puntuaciones obtenidas en el IRI, correspondientes a las cuatro dimensiones de

Tabla 2. Valores medios en el Cuestionario de Apego Adulto

\begin{tabular}{lccccccc}
\hline \multicolumn{2}{c}{$\begin{array}{c}\text { Agresores } \\
(\mathrm{N}=90)\end{array}$} & \multicolumn{2}{c}{$\begin{array}{c}\text { Controles } \\
(\mathrm{N}=94)\end{array}$} \\
\hline & $\mathrm{M}$ & $\mathrm{DT}$ & $\mathrm{M}$ & $\mathrm{DT}$ & $t$ & $p$ & $d$ \\
Escala 1 & 33.60 & 10.9 & 37.97 & 10.0 & -2.506 & 0.013 & -0.42 \\
Escala 2 & 28.41 & 8.8 & 30.61 & 9.2 & -1.481 & 0.141 & - \\
Escala 3 & 42.56 & 6.4 & 42.08 & 6.4 & 0.452 & 0.652 & - \\
Escala 4 & 16.38 & 5.3 & 18.00 & 6.5 & -1.683 & 0.095 & - \\
\hline
\end{tabular}

Escala 1: baja autoestima, necesidad de aprobación y miedo al rechazo; Escala 2: resolución hostil de conflictos, rencor y posesividad; Escala 3: expresión de sentimientos y comodidad con las relaciones; Escala 4: autosuficiencia emocional e incomodidad con la intimidad.

Tabla 3. Puntuaciones según los baremos de Melero \& Cantero (2008)

\begin{tabular}{lllll}
\hline & \multicolumn{2}{c}{ Agresores } & Controles & Valoración \\
\hline & Centil & Valoración & $40-60$ & Moderado \\
Escala 1 & $25-39$ & Bajo/Mod. & $40-60$ & Moderado \\
Escala 2 & $40-60$ & Moderado & $40-60$ & Moderado \\
Escala 3 & $61-74$ & Mod./Alto & $61-74$ & Mod./Alto \\
Escala 4 & $40-60$ & Moderado &
\end{tabular}

Tabla 4. Puntuaciones en dimensiones empáticas del IRI

\begin{tabular}{lcccccc}
\hline & \multicolumn{2}{c}{$\begin{array}{c}\text { Agresores } \\
(\mathrm{N}=90)\end{array}$} & \multicolumn{3}{c}{$\begin{array}{c}\text { Controles } \\
(\mathrm{N}=94)\end{array}$} \\
\hline & $\mathrm{M}$ & $\mathrm{DT}$ & $\mathrm{M}$ & $\mathrm{DT}$ & $\mathrm{t}$ & 0.963 \\
\hline Toma de perspectiva & 24.53 & 4.8 & 21.72 & 5.8 & -0.046 & -0.398 \\
Fantasía & 18.87 & 5.0 & 17.49 & 5.6 & -0.503 & 0.692 \\
Preocupación empática & 26.23 & 5.0 & 22.45 & 7.4 & 0.616 \\
Malestar personal & 15.34 & 4.5 & 13.70 & 5.6 & -0.762 & 0.447 \\
\hline
\end{tabular}


Tabla 5. Puntuaciones en Autoestima

\begin{tabular}{|c|c|c|c|c|c|c|}
\hline & \multicolumn{2}{|c|}{$\begin{array}{c}\text { Agresores } \\
(\mathrm{N}=90)\end{array}$} & \multicolumn{2}{|c|}{$\begin{array}{c}\text { Controles } \\
(\mathrm{N}=94)\end{array}$} & \multirow[b]{2}{*}{$t / \chi^{2}$} & \multirow[b]{2}{*}{$p$} \\
\hline & $\mathrm{M}$ & DT & $\mathrm{M}$ & DT & & \\
\hline Puntuación total & 33.33 & 4.9 & 32.48 & 3.95 & 0.993 & 0.322 \\
\hline \multicolumn{7}{|l|}{ Categorías } \\
\hline $\begin{array}{l}\text { Baja } \\
\text { Media } \\
\text { Alta }\end{array}$ & \multicolumn{2}{|c|}{$\begin{array}{c}8.2 \% \\
16.5 \%\end{array}$} & & & 2.93 & 0.231 \\
\hline
\end{tabular}

Tabla 6. Variables sociodemográficas de los dos grupos de agresores

\begin{tabular}{|c|c|c|c|c|}
\hline & $\begin{array}{c}\text { Grupo } 1 \\
(\mathrm{n}=50 ; 55.6 \%)\end{array}$ & $\begin{array}{c}\text { Grupo } 2 \\
(n=40 ; \\
44.4 \%)\end{array}$ & $\mathrm{z} / \chi^{2}$ & $p$ \\
\hline $\operatorname{Edad}(M)$ & 35 & 38 & -1.64 & 0.102 \\
\hline \multicolumn{5}{|l|}{ Nacionalidad } \\
\hline Español & $66 \%$ & $72.5 \%$ & 0.591 & 0.442 \\
\hline Extranjero & $34.0 \%$ & $27.5 \%$ & & \\
\hline \multicolumn{5}{|l|}{ Estudios } \\
\hline Primarios & $28.3 \%$ & $18.4 \%$ & & \\
\hline Secundarios & $58.7 \%$ & $57.9 \%$ & 2.211 & 0.530 \\
\hline Formación Profesional & $6.5 \%$ & $13.2 \%$ & & \\
\hline Universitarios & $6.5 \%$ & $10.5 \%$ & & \\
\hline
\end{tabular}

Tabla 7. Comparación en variables afectivas entre grupos de agresores y respecto al grupo de control

\begin{tabular}{|c|c|c|c|c|c|c|c|c|c|c|c|c|}
\hline & \multicolumn{2}{|c|}{$\begin{array}{c}\text { Grupo } 1 \\
(\mathrm{n}=50 ; 55.6 \%)\end{array}$} & \multicolumn{2}{|c|}{$\begin{array}{c}\text { Grupo } 2 \\
(n=40 ; \\
44.4 \%)\end{array}$} & \multicolumn{2}{|c|}{$\begin{array}{c}\text { Grupo } \\
\text { Control } \\
(\mathrm{N}=94)\end{array}$} & \multicolumn{2}{|c|}{$\begin{array}{c}\text { Diferencias } \\
\text { entre agresores }\end{array}$} & \multicolumn{2}{|c|}{$\begin{array}{c}\text { Grupo } 1 \\
\text { vs. } \\
\text { Control }\end{array}$} & \multicolumn{2}{|c|}{$\begin{array}{c}\text { Grupo } 2 \\
\text { vs. } \\
\text { Control }\end{array}$} \\
\hline & $X$ & $D T$ & $X$ & $D T$ & $X$ & $D T$ & $z$ & $d$ & $z$ & $d$ & $z$ & $d$ \\
\hline \multicolumn{13}{|l|}{ Apego adulto } \\
\hline Escala 1 & 39.89 & 8.9 & 25.45 & 7.1 & 37.97 & 10.0 & $-6.34 * * *$ & 1.79 & -1.14 & - & $-5.76 * * *$ & -1.44 \\
\hline Escala 2 & 33.47 & 6.8 & 21.89 & 6.4 & 30.61 & 9.2 & $-6.32 * * *$ & 1.75 & $-2.08^{*}$ & 0.35 & $-4.64 * * *$ & -1.10 \\
\hline Escala 3 & 41.87 & 6.3 & 43.50 & 6.6 & 42.08 & 6.4 & -1.25 & - & -0.11 & - & -1.17 & - \\
\hline Escala 4 & 18.38 & 5.1 & 14.00 & 4.6 & 18.00 & 6.5 & $-3.82 * * *$ & 0.90 & -0.42 & - & $-3.04 * *$ & -0.71 \\
\hline Autoestima & 31.94 & 5.2 & 35.05 & 4.0 & 32.48 & 3.9 & $-2.72 * *$ & -0.67 & -0.56 & - & $-3.25 * * *$ & 0.65 \\
\hline \multicolumn{13}{|l|}{ Empatía } \\
\hline $\begin{array}{r}\text { Toma de } \\
\text { perspectiva }\end{array}$ & 23.26 & 4.0 & 26.13 & 5.4 & 21.72 & 5.8 & $-2.78 * *$ & -0.61 & -1.61 & - & -1.73 & - \\
\hline Fantasía & 19.49 & 5.1 & 17.79 & 4.8 & 17.49 & 5.6 & -1.57 & - & -0.39 & - & -1.27 & - \\
\hline $\begin{array}{r}\text { Preocupación } \\
\text { empática }\end{array}$ & 26.64 & 4.8 & 25.66 & 5.3 & 22.45 & 7.4 & -1.25 & - & -0.22 & - & -0.94 & - \\
\hline $\begin{array}{l}\text { Malestar } \\
\text { personal }\end{array}$ & 16.77 & 4.6 & 13.39 & 3.8 & 13.70 & 5.6 & $-3.58 * * *$ & 0.80 & -1.02 & - & $-2.74 * *$ & -0.06 \\
\hline
\end{tabular}

empatía. Las diferencias no son significativas en ninguna de las escalas. Respecto a la adaptación española, la muestra de agresores se sitúa en el centil 85 o por encima en las cuatro dimensiones, y el grupo de control en el centil 80 o superior en todas ellas (aunque dichos valores corresponden a población adolescente).

\section{Autoestima}

Tal como se observa en la tabla 5 , no existen diferencias entre el grupo de agresores y el grupo de control en la Escala de Autoestima. Una proporción superior al $75 \%$ en ambas muestras presenta una autoestima elevada (puntuación $\geq 30$ ). 


\section{Análisis de clusters}

La muestra de agresores se ha clasificado, mediante análisis de clusters, en dos grupos (ver tabla 6). El objetivo de este paso ha sido ver si era posible establecer diferencias entre los agresores que puedan presentar un estilo afectivo más problemático respecto a otros agresores más "saludables" y respecto al grupo de control proveniente de la población general.

En la tabla 7 se describen las características afectivas de los dos grupos de agresores establecidos a partir de sus puntuaciones en las tres escalas. El grupo 1 presenta significativamente más necesidad de aprobación y miedo al rechazo (escala 1 del CAA), así como resolución hostil y distanciamiento emocional; menor autoestima (aunque la puntuación media equivale a la categoría de autoestima elevada); y mayor malestar empático. El grupo 2 destaca en expresión de sentimientos y comodidad con las relaciones, aunque sin diferencias significativas respecto al grupo 1, tiene significativamente más autoestima y menor malestar empático (sin diferencias en el resto de variables de empatía). Por tanto, el grupo 2 mostraría un estilo afectivo más "saludable".

Respecto a la comparación entre subgrupos de agresores y el grupo de control, los agresores del grupo 1 solo presentarían diferencias estadísticamente significativas en la escala 2 del CAA, mostrando así mayor resolución hostil de conflictos, rencor y posesividad. El grupo 2 mostraría más diferencias significativas respecto al grupo de control, con puntuaciones significativamente menores en la escalas del CAA 1 (baja autoestima, necesidad de aprobación y miedo al rechazo), 2 (resolución hostil de conflictos, rencor y posesividad) y 4 (autosuficiencia emocional e incomodidad con la intimidad), todas ellas variables relacionadas con un apego no seguro. Asimismo presentan significativamente más autoestima o menor malestar empático (una variable relacionada con un malestar egocéntrico).

\section{Discusión}

Los resultados obtenidos no han permitido confirmar las hipótesis de las que se partía. Así pues, se esperaba un estilo afectivo diferente en los agresores respecto al grupo de control, con una menor empatía, una autoestima más baja y estilos de apego problemáticos, como el inseguro o el ansioso. Junto a ello, se esperaba poder diferenciar distintos tipos de agresores a partir de estas variables. Pese a que el establecimiento de dos grupos ha sido posible, las diferencias respecto al grupo de control no han sido las esperadas.

Respecto a la variable apego adulto, los resultados no han permitido establecer diferencias congruentes con investigaciones previas entre agresores y controles (Fournier et al., 2011; Lawson \& Brossart, 2009; Mauricio \& López, 2009; Mikulincer \& Shaver, 2007; Tweed \& Dutton, 1998).
Con las puntuaciones obtenidas no se ha podido clasificar al grupo de control en ningún tipo de apego y los agresores se ubicarían en puntuaciones propias del apego seguro (para dos dimensiones) y el apego preocupado o alejado (para cuatro dimensiones). Como ya se ha comentado, no existen estudios previos en los que se haya utilizado el CAA para valorar agresores de pareja, por lo que tampoco es posible comparar las puntuaciones.

Los resultados obtenidos en autoestima van en contra de la hipótesis predominante a nivel académico de que la conducta violenta se relaciona con una baja autoestima. Sin embargo, son congruentes con las propuestas más recientes que ponen en entredicho esta afirmación y señalan que la autoestima no tiene un efecto independiente sobre la violencia (Bushman et al., 2009; Maples et al., 2010; Ostrowsky, 2010; Thomaes \& Bushman, 2011). Las hipótesis que podrían explicar la presencia de una elevada autoestima entre agresores podrían ser las siguientes: 1) la autoestima no está asociada con la conducta violenta y realmente no existen diferencias entre las personas violentas y las que no lo son (Ostrowsky, 2010); 2) la escala utilizada es poco sensible o específica y no evalúa las posibles dimensiones de la autoestima que podrían explicar distintas conductas (Ostrowsky, 2010; Sakellaropoulo \& Baldwin, 2007; Walker \& Bright, 2009; Webster et al., 2007); y 3) los resultados podrían ser reflejo de la existencia de una falsa autoestima inflada en los agresores, con un trasfondo de baja autoestima y rasgos narcisistas (Bushman et al., 2009; Maples et al., 2010; Salmivalli, 2001; Walker \& Bright, 2009). Las puntuaciones en autoestima podrían considerarse similares a las de otros estudios con muestras españolas, con una autoestima media alta (Echeburúa, Fernández-Montalvo \& Amor, 2003; Echeburúa et al., 2006), aunque la puntuación sería superior a la obtenida en medidas post-tratamiento (Echeburúa \& Fernández-Montalvo, 2009).

Por último, la ausencia de diferencias en empatía, pese a ser un resultado poco esperado, es congruente con investigaciones recientes. Así, se afirma que la empatía depende del contexto o de la víctima y no es un rasgo estable (Day et al., 2011; Marshall et al., 2009; Martínez, Redondo, Pérez \& García, 2008), y que realmente no es una variable que permita diferenciar agresores de grupos normativos ni que su tratamiento pueda reducir la reincidencia (Day et al., 2010; Huss \& Ralston, 2008; Jolliffe \& Farrington, 2004). Tal vez por eso, las tasas de psicopatía son más bajas de las esperadas en muestras de agresores encarcelados (Echeburúa \& Fernández-Montalvo, 2007; Fernández-Montalvo \& Echeburúa, 2008). Respecto a otras muestras de agresores, nuestro grupo presenta puntuaciones similares en los dos primeros factores, algo superiores en el factor 3 e intermedias en el 4 (Boira \& Tomás, 2011; Covell et al., 2007).

La investigación presenta una serie de limitaciones que deben ser tenidas en cuenta a la hora de valorar los 
resultados. En primer lugar, no se ha tomado en cuenta la deseabilidad social, una variable que puede influir directamente en las puntuaciones obtenidas (Novo, Fariña, Seijo \& Arce, 2012). La escala de deseabilidad social de Marlowe \& Crowne se había incluido en el protocolo de evaluación de los agresores, pero su ausencia en el grupo de control ha hecho que el análisis de la variable no se haya incorporado a este estudio. Por otro lado, pese a que se han descrito las variables en los agresores y se han establecido dos grupos a partir de estas, no se ha determinado la asociación entre un peor "funcionamiento afectivo" y una gravedad diferencial de la violencia, por ejemplo en las respuestas dadas en la CTS-2. Este aspecto podría resultar de gran interés para investigaciones futuras.

En definitiva, los resultados no permiten apoyar la posible utilidad de incluir este tipo de variables en los programas de tratamiento (en línea con estudios previos de Day et al., 2010), pese a que serán necesarias futuras replicaciones de los resultados para poder alcanzar esta conclusión. Uno de los principales problemas en este ámbito de investigación es la fiabilidad de las medidas de evaluación (Jolliffe \& Farrington, 2004; Ostrowsky, 2010; Walker \& Bright, 2009; Walker \& Knauer, 2011). Habitualmente, los instrumentos se han validado en muestras de estudiantes y podemos estar haciendo atribuciones sobre la mayor o menor presencia de una determinada variable cuando las muestras no son comparables. Pese al interés que supone comprender mejor el mecanismo mediante el cual las variables afectivas, como la empatía, el apego y la autoestima, están relacionadas con la agresión para diseñar estrategias de intervención adecuadas, antes resulta imprescindible establecer puntuaciones específicas para muestras concretas que nos permitan valorar con precisión los resultados obtenidos.

\section{Referencias}

Alonso-Arbiol, I., Balluerka, N., \& Shaver, P. R. (2007). A spanish version of the experiences in close relationships (ECR) adult attachment questionnaire. Personal Relationships, 14, 45-63.

Alonso-Arbiol, I., Shaver, P. R., \& Yarnoz, S. (2002). Insecure attachment, gender roles, and interpersonal dependency in the Basque Country. Personal Relationships, 9, 479-490.

Amor, P. J., Echeburúa, E., \& Loinaz, I. (2009). ¿Se puede establecer una clasificación tipológica de los hombres violentos contra su pareja? International Journal of Clinical and Health Psychology, 9, 519-539.

Ansbro, M. (2008). Using attachment theory with offenders. Probation Journal, 55, 231-244.

Babcock, J. C., Jacobson, N. S., Gottman, J. M., \& Yerington, T. P. (2000). Attachment, emotional regulation, and the function of marital violence: Differences between secure, preoccupied, and dismissing violent and nonviolent husbands. Journal of Family Violence, 15, 391-409.

Beech, A. R., \& Mitchell, I. J. (2009). Attachment difficulties. En McMurran M., \& Howard R. (Eds.), Personality, personality disorder, and violence: An evidence-based approach (pp. 213-228). Chichester, UK: John Wiley, \& Sons.

Boira, S., López, Y., Tomás, L., \& Gaspar, A. (2010). Evaluación cualitativa de un programa de intervención psicológica con hombres violentos dentro de la pareja. Acciones e Investigaciones Sociales, 28, 135-156.
Boira, S., \& Tomás, L. (2011). Características psicológicas y motivación para el cambio en hombres condenados por violencia contra la pareja. International Journal of Psychological Research, 4, 48-56.

Bowen, E. (2011). The rehabilitation of partner-violent men. Chichester: Wiley-Blackwell.

Bushman, B. J., Baumeister, R. F., Thomaes, S., Ryu, E., Begeer, S., \& West, S. G. (2009). Looking again, and harder, for a link between low self-esteem and aggression. Journal of Personality, 77, 427-446.

Cale, E. M., \& Lilienfeld, S. O. (2006). Psychopathy factors and risk for aggressive behavior: A test of the "threatened egotism" hypothesis. Law and Human Behavior, 30, 51-74.

Covell, C. N., Huss, M. T., \& Langhinrichsen-Rohling, J. (2007). Empathic deficits among male batterers: A multidimensional approach. Journal of Family Violence, 22, 165-174.

Davis, M. H. (1980). A multidimensional approach to individual differences in empathy. JSAS Catalog of Selected Documents in Psychology, 10,85 .

Davis, M. H. (1996). Empathy: A social psychological approach. Boulder, CO: Westview Press.

Day, A., Casey, S., \& Gerace, A. (2010). Interventions to improve empathy awareness in sexual and violent offenders: Conceptual, empirical, and clinical issues. Aggression and Violent Behavior, 15, 201-208.

Day, A., Mohr, P., Howells, K., Gerace, A., \& Lim, L. (2011). The role of empathy in anger arousal in violent offenders and university students. International Journal of Offender Therapy and Comparative Criminology. Advance online publication doi: 10.1177/0306624x11431061

Dick, G. (2004). Men's relationships with their fathers: Comparing men who batter women with non-violent men. Journal of Emotional Abuse, 4, 61-84.

Dobash, R. E., Dobash, R. P., Cavanagh, K., \& Lewis, R. (2000). Changing violent men. London: Sage.

Dutton, D. G. (1988). Profiling of wife assaulters: Preliminary evidence for a trimodal analysis. Violence and Victims, 3, 5-29.

Dutton, D. G. (2007). The abusive personality. Violence and control in intimate relationships ( $2^{\mathrm{a}}$ ed.). New York: The Guilford Press.

Dutton, D. G. (2008). Blended behavior therapy for intimate violence. En A. C. Baldry, \& F. W. Winkel (Eds.), Intimate partner violence prevention and intervention: The risk assessment and management approach (pp. 133-146). New York: Nova Science Publishers.

Dutton, D. G. (2011). Attachment and violence: An anger born of fear. En P. R. Shaver, \& M. Mikulincer (Eds.), Human aggression and violence. Causes, manifestations, and consequences (pp. 259-275). Washington, DC: American Psychological Association.

Dutton, D. G., \& Golant, S. K. (1997). El golpeador. Un perfil psicológico. Buenos Aires: Paidós.

Dutton, D. G., Saunders, K., Starzomski, A., \& Bartholomew, K. (1994). Intimacy-anger and insecure attachment as precursors of abuse in intimate relationships. Journal of Applied Social Psychology, 24, 1367-1386

Echeburúa, E., \& Fernández-Montalvo, J. (2007). Male batterers with and without psychopathy: An exploratory study in spanish prisons. International Journal of Offender Therapy and Comparative Criminology, 51, 254-263.

Echeburúa, E., \& Fernández-Montalvo, J. (2009). Evaluación de un programa de tratamiento en prisión de hombres condenados por violencia grave contra la pareja. International Journal of Clinical and Health Psychology, 9, 5-20.

Echeburúa, E., Fernández-Montalvo, J., \& Amor, P. J. (2003). Psychopathological profile of men convicted of gender violence: A study in the prisons of Spain. Journal of Interpersonal Violence, 18, 798-812.

Echeburúa, E., Fernández-Montalvo, J., \& Amor, P. J. (2006). Psychological treatment of men convicted of gender violence: A pilot study in Spanish prisons. International Journal of Offender Therapy and Comparative Criminology, 50, 57-70.

Echeburúa, E., Sarasua, B., Zubizarreta, I., \& de Corral, P. (2009). Evaluación de la eficacia de un tratamiento cognitivo-conductual para hombres violentos contra la pareja en un marco comunitario: Una experiencia 
de 10 años (1997-2007). International Journal of Clinical and Health Psychology, 9, 199-217.

Fernández-Montalvo, J., \& Echeburúa, E. (2008). Trastornos de la personalidad y psicopatía en hombres condenados por violencia grave contra la pareja. Psicothema, 20, 193-198.

Fernández-Pinto, I., López-Pérez, B., \& Márquez, M. (2008). Empatía: Medidas, teorías y aplicaciones en revisión. Anales de Psicología, 24, 284-298.

Forbes, D., \& Reilly, S. (2011). 'Using attachment theory with offenders' by Maria Ansbro. Probation Journal, 58, 167-171.

Fournier, B., Brassard, A., \& Shaver, P. R. (2011). Adult attachment and male aggression in couple relationships: The demand-withdraw communication pattern and relationship satisfaction as mediators. Journal of Interpersonal Violence, 26, 1982-2003.

Gondolf, E. W. (2002). Batterer Intervention Systems: Issues, Outcomes, and Recommendations.

Holtzworth-Munroe, A., \& Meehan, J. C. (2004). Typologies of men who are maritally violent - Scientific and clinical implications. Journal of Interpersonal Violence, 19, 1369-1389.

Holtzworth-Munroe, A., Meehan, J. C., Herron, K., Rehman, U., \& Stuart, G. L. (2000). Testing the Holtzworth-Munroe and Stuart (1994) batterer typology. Journal of Consulting and Clinical Psychology, 68, 1000-1019.

Holtzworth-Munroe, A., Meehan, J. C., Herron, K., Rehman, U., \& Stuart, G. L. (2003). Do subtypes of maritally violent men continue to differ over time? Journal of Consulting and Clinical Psychology, 71, 728-740.

Holtzworth-Munroe, A., \& Stuart, G. L. (1994). Typologies of male batterers: Three subtypes and the differences among them. Psychological Bulletin, 116, 476-497.

Holtzworth-Munroe, A., Stuart, G. L., \& Hutchinson, G. (1997). Violent versus nonviolent husbands: Differences in attachment patterns, dependency, and jealousy. Journal of Family Psychology, 11, 314-331.

Hollin, C. R. (Ed.), (2003). The essential handbook of offender assessment and treatment. Chichester: John Wiley, \& Sons.

Huss, M. T., \& Ralston, A. (2008). Do batterer subtypes actually matter? Treatment completion, treatment response, and recidivism across a batterer typology. Criminal Justice and Behavior, 35, 710-724.

Jolliffe, D., \& Farrington, D. P. (2004). Empathy and offending: A systematic review and meta-analysis. Aggression and Violent Behavior, 9, 441-476

Jolliffe, D., \& Farrington, D. P. (2007). Examining the relationship between low empathy and self-reported offending. Legal and Criminological Psychology, 12, 265-286.

Jones, D. (2008). Understanding criminal behaviour: Psychosocial approaches to criminality. Oxon: William Publishing.

Kirkpatrick, L. A., Waugh, C. E., Valencia, A., \& Webster, G. D. (2002). The functional domain specificity of self-esteem and the differential prediction of aggression. Journal of Personality and Social Psychology, 82, 756-767.

Lawson, D. M. (2008). Attachment, interpersonal problems, and family of origin functioning: Differences between partner violent and nonpartner violent men. Psychology of Men and Masculinity, 9, 90-105.

Lawson, D. M., Barnes, A. D., Madkins, J. P., \& Francois-Lamonte, B. M. (2006). Changes in male partner abuser attachment styles in group treatment. Psychotherapy, 43, 232-237.

Lawson, D. M., \& Brossart, D. F. (2009). Attachment, interpersonal problems, and treatment outcome in group therapy for intimate partner violence. Psychology of Men \& Masculinity, 10, 288-301.

Loinaz, I. (2011a). Clasificación de agresores de pareja en prisión. Implicaciones terapéuticas y de gestión del riesgo. En Intervención con agresores de violencia de género (pp. 153-276). Barcelona: Centro de Estudios Jurídicos y Formación Especializada, Generalitat de Catalunya.

Loinaz, I. (2011b). Estudo de tipologias de agressors conjugais em prisões. Ousar Integrar-Revista de Reinserção Social e Prova, 9, 23-34.

Loinaz, I., Echeburúa, E., Ortiz-Tallo, M., \& Amor, P. J. (2012). Propiedades psicométricas de la Conflict Tactics Scales (CTS-2) en una muestra española de agresores de pareja. Psicothema, 24, 142-148.
Loinaz, I., Echeburúa, E., \& Torrubia, R. (2010). Tipología de agresores contra pareja en prisión. Psicothema, 22, 106-111.

Maples, J. L., Miller, J. D., Wilson, L. F., Seibert, L. A., Few, L. R., \& Zeichner, A. (2010). Narcissistic personality disorder and self-esteem: An examination of differential relations with self-report and laboratorybased aggression. Journal of Research in Personality, 44, 559-563.

Marshall, W. L., Marshall, L. E., Serran, G. A., \& O’Brien, M. D. (2009). Self-esteem, shame, cognitive distortions and empathy in sexual offenders: Their integration and treatment implications. Psychology, Crime and Law, 15, 217-234.

Martínez, M., Redondo, S., Pérez, M., \& García, C. (2008). Empatía en una muestra española de delincuentes sexuales. Psicothema, 20, 199-204.

Mauricio, A. M., \& Gormley, B. (2001). Male perpetration of physical violence against female partners: The interaction of dominance needs and attachment insecurity. Journal of Interpersonal Violence, 16, 1066-1081.

Mauricio, A. M., \& López, F. G. (2009). A latent classification of male batterers. Violence and Victims, 24, 419-438.

Mayseless, O. (1991). Adult attachment patterns and courtship violence. Family Relations, 40, 21-28.

McMurran, M., \& Howard, R. C. (Eds.). (2009). Personality, personality disorder and violence. Chichester, UK: John Wiley, \& Sons.

McPhedran, S. (2009). A review of the evidence for associations between empathy, violence, and animal cruelty. Aggression and Violent Behavior, 14, 1-4.

Melero, R., \& Cantero, M. J. (2008). Los estilos afectivos en la población española: Un cuestionario de evaluación del apego adulto. Clínica y Salud, 19, 83-100.

Meloy, J. R. (2003). Pathologies of attachment, violence, and criminality. En A. M. Goldstein (Ed.), Handbook of Psychology (Vol. 11, Forensic Psychology, pp. 509-526). New York: Wiley.

Mestre, M., Frías, M. D., \& Samper, P. (2004). La medida de la empatía: análisis del Interpersonal Reactivity Index. Psicothema, 16, 255-260.

Mikulincer, M., \& Shaver, P. R. (2007). Attachment in adulthood: Structure, dynamics, and change. New York, NY: The Guilford Press.

Mikulincer, M., \& Shaver, P. R. (2011). Attachment, anger, and aggression. En P. R. Shaver, \& M. Mikulincer (Eds.), Human aggression and violence. Causes, manifestations, and consequences (pp. 241-257). Washington, DC: American Psychological Association.

Millana, L. (2011). Intervention programs for Spanish inmate aggressors convicted of domestic violence. The Open Criminology Journal, 4, 91-101.

Novo, M., Fariña, F., Seijo, D., \& Arce, R. (2012). Assessment of a community rehabilitation programme in convicted male intimate-partner violence offenders. International Journal of Clinical and Health Psychology, 12, 219-234.

Ostrowsky, M. K. (2010). Are violent people more likely to have low selfesteem or high self-esteem? Aggression and Violent Behavior, 15, 69-75.

Owen, T., \& Fox, S. (2011). Experiences of shame and empathy in violent and non-violent young offenders. Journal of Forensic Psychiatry and Psychology, 22, 551-563.

Palmer, E. J. (2005). The relationship between moral reasoning and aggression, and the implications for practice. Psychology, Crime and Law, 11, 353-361.

Papadakaki, M., Tzamalouka, G. S., Chatzifotiou, S., \& Chliaoutakis, J. (2009). Seeking for risk factors of Intimate Partner Violence (IPV) in a greek national sample: The role of self-esteem. Journal of Interpersonal Violence, 24, 732-750.

Renzetti, C. M., \& Edleson, J. L. (2008). Encyclopedia of interpersonal violence. Thousand Oaks, CA: Sage.

Rosenberg, M. (1965). Society and the Adolescent Self-image. Princeton, NJ: Princeton University Press.

Ross, T., \& Pfäfflin, F. (2007). Attachment and interpersonal problems in a prison environment. Journal of Forensic Psychiatry and Psychology, 18, 90-98.

Sakellaropoulo, M., \& Baldwin, M. W. (2007). The hidden sides of selfesteem: Two dimensions of implicit self-esteem and their relation to narcissistic reactions. Journal of Experimental Social Psychology, 43, 995-1001. 
Salmivalli, C. (2001). Feeling good about oneself, being bad to others? Remarks on self-esteem, hostility, and aggressive behavior. Aggression and Violent Behavior, 6, 375-393.

Sandstrom, M. J., \& Jordan, R. (2008). Defensive self-esteem and aggression in childhood. Journal of Research in Personality, 42, 506-514.

Saunders, D. G. (1992). A typology of men who batter: Three types derived from cluster analysis. American Journal of Orthopsychiatry, 62, 264-275.

Shaver, P. R., \& Mikulincer, M. (2011). Human aggression and violence. Causes, manifestations, and consequences. Washington, DC: American Psychological Association.

Taylor, L. D., Davis-Kean, P., \& Malanchuk, O. (2007). Self-esteem, academic self-concept, and aggression at school. Aggressive Behavior, $33,130-136$.

Thomaes, S., \& Bushman, B. J. (2011). Mirror, mirror, on the wall, who's the most aggressive of them all? Narcissism, self-esteem, and aggression. En P. R. Shaver, \& M. Mikulincer (Eds.), Human aggression and violence. Causes, manifestations, and consequences (pp. 203-219). Washington, DC: American Psychological Association.
Thomaes, S., Bushman, B. J., Stegge, H., \& Olthof, T. (2008). Trumping shame by blasts of noise: Narcissism, self-esteem, shame, and aggression in young adolescents. Child development, 79, 1792-1801.

Tweed, R. G., \& Dutton, D. G. (1998). A comparison of impulsive and instrumental subgroups of batterers. Violence and Victims, 13, 217-230.

Vázquez, J., Jiménez, R., \& Vázquez-Morejón, R. (2004). Escala de Autoestima de Rosenberg: fiabilidad y validez en población clínica española. Apuntes de Psicología, 22, 247-255.

Walker, J. S., \& Bright, J. A. (2009). False inflated self-esteem and violence: A systematic review and cognitive model. Journal of Forensic Psychiatry and Psychology, 20, 1-32.

Walker, J. S., \& Knauer, V. (2011). Humiliation, self-esteem and violence. Journal of Forensic Psychiatry \& Psychology, 22, 724-741.

Webster, G. D., Kirkpatrick, L. A., Nezlek, J. B., Smith, C. V., \& Paddock, E. L. (2007). Different slopes for different folks: Self-esteem instability and gender as moderators of the relationship between self-esteem and attitudinal aggression. Self and Identity, 6, 74-94 